\title{
Improving the quality of reporting randomized controlled trials in cardiothoracic surgery: The way forward
}

\author{
Ravindranath Tiruvoipati, FRCSEd, ${ }^{\text {a }}$ Sabapathy P. Balasubramanian, FRCSEd, ${ }^{\text {b }}$ Gnaneswar Atturu, MRCS, ${ }^{\text {a }}$
} Giles J. Peek, MD, FRCS (C Th), and Diana Elbourne, $\mathrm{PhD}^{\mathrm{c}}$

See related articles on pages $229,241,243,245,247$, and 249.
From the Departments of Cardiac Surgery and ECMO, Glenfield Hospital, Leicester, Leicester, United Kingdoma; Academic Unit of Surgical Oncology, University of Sheffield, Sheffield, United Kingdom ${ }^{\text {b }}$; and London School of Hygiene and Tropical Medicine, University of London, London, United Kingdom. ${ }^{c}$

Received for publication June 27, 2005; revisions received Sept 13, 2005; accepted for publication Oct 28, 2005.

Address for reprints: Ravindranath Tiruvoipati, FRCSEd, Department of ECMO, Glenfield Hospital, Groby Rd, Leicester, LE3 9QP, UK (E-mail: rtiruvoipati@ yahoo.co.uk).

J Thorac Cardiovasc Surg 2006;132:233-40

$0022-5223 / \$ 32.00$

Copyright (c) 2006 by The American Association for Thoracic Surgery

doi:10.1016/j.jtcvs.2005.10.056
Objective: To evaluate the quality of reporting of randomized controlled trials in cardiothoracic surgery, to identify factors associated with good reporting quality, and to assess the awareness of the Consolidated Standards for Reporting of Trials statement and ascertain the views of authors reporting randomized controlled trials on the difficulties in conducting randomized controlled trials and the possible ways to further improve the reporting quality of randomized controlled trials in cardiothoracic surgery.

Methods: Randomized controlled trials of cardiothoracic surgery published in principal cardiothoracic and 4 general medical journals in 2003 were included. The quality of reporting of randomized controlled trials was assessed by using allocation concealment, the Jadad score, and a Consolidated Standards for Reporting of Trials checklist devised for the purpose. A questionnaire survey of authors reporting randomized controlled trials in principal cardiothoracic journals in 2003 was conducted.

Results: The overall reporting quality of the 64 randomized controlled trials included in the analysis was suboptimal as assessed by the 3 methods adopted. Most of the authors (63.5\%) were not aware of the Consolidated Standards for Reporting of Trials statement; however, awareness was not associated with reporting quality. More than $65 \%$ of the authors responded that conducting randomized controlled trials in surgical specialties was difficult, and the main difficulties were blinding and obtaining a large-enough sample size to detect statistically significant differences. Fifty-four percent of the authors responded that endorsement of the Consolidated Standards for Reporting of Trials statement by the cardiothoracic journals may improve the reporting quality.

Conclusions: The quality of reporting randomized controlled trials in cardiothoracic surgery is suboptimal. Endorsement of the Consolidated Standards for Reporting of Trials statement by the cardiothoracic journals may improve the quality of reporting.

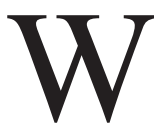
ell-designed, properly conducted, and clearly reported randomized controlled trials (RCTs) provide the best evidence for health care professionals to make appropriate clinical decisions about the relative effectiveness of different interventions. ${ }^{1}$ High-quality reports are likely to improve the interpretation of RCTs and minimize biased conclusions. ${ }^{2}$ There is, however, strong evidence to indicate that the quality of the reporting of RCTs in the medical literature is less than optimal. ${ }^{3,4}$ To overcome such problems in reporting, the Consolidated Standards for Reporting of Trials (CONSORT) Group developed the CONSORT statement ${ }^{5}$ in 1996, followed by a revised version ${ }^{3}$ in 2001 , which was accompanied by a longer document providing further elaboration and explanation. ${ }^{1}$ The CONSORT statement has been widely supported and has been translated into several languages to facilitate awareness and dissemination. ${ }^{6,7}$ The CONSORT 


\section{Abbreviations and Acronyms \\ CONSORT $=$ Consolidated Standards for Reporting of Trials \\ RCT $=$ randomized controlled trial}

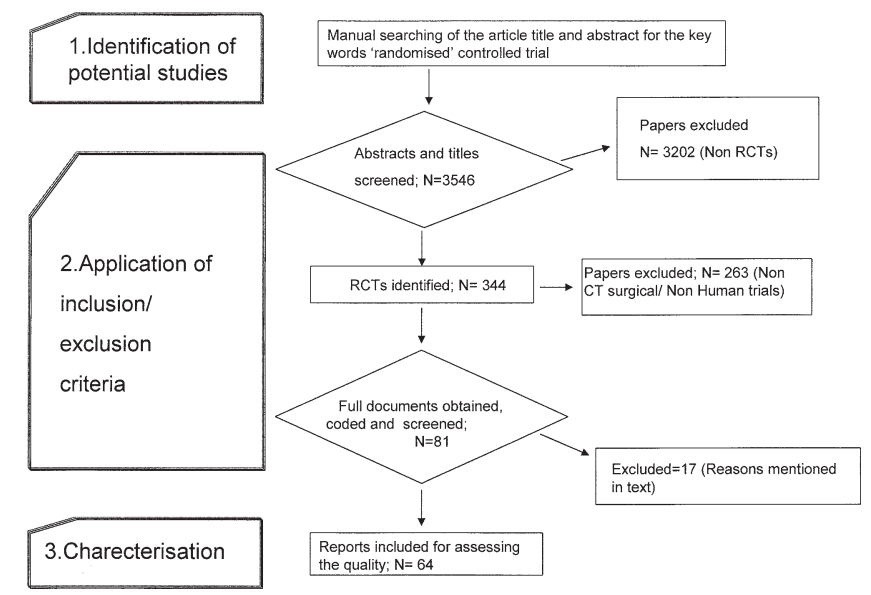

Figure 1. Filtering of articles from searching to reports included in analysis. CT, Cardiothoracic.

choosing the these general medical journals (with a high impact factor) for the study. The search strategy (Figure 1) involved manual screening of the titles and abstracts of all the articles published in these journals in 2003 for the key words "randomized controlled trial." Full reports were obtained for studies that seemed to meet the criteria or where there was insufficient information in the title and abstract. Studies were excluded from assessment of their reporting quality if they were predominantly trial design articles, had predominantly nonclinical outcomes as end points, were not the main report of the trial results, were not related to cardiothoracic surgical practice, did not involve human subjects, were cluster-randomized trials, or were not truly randomized. We also initially excluded trials if there had been a previous report of their design and methods before the publication of revised CONSORT statement, but we subsequently conducted sensitivity analyses to explore the effect of this exclusion.

"Instructions to Authors" sections of all the journals were accessed (in November 2003 and in June 2005) to find out whether they endorsed the CONSORT statement. Specific descriptive characteristics for each article (the number of authors, number of centers involved, involvement of a statistician/epidemiologist, source of funding [if any], and country of study) were noted by one observer (G.A.), who then masked the articles so that the other reviewers were not aware in which journal the article was published.

Part of the methodology used to assess the quality of the RCT reports was similar to that described by Moher and colleagues. ${ }^{15}$ The reporting of allocation concealment was assessed as adequate, inadequate, or unclear as described by Schulz and colleagues. ${ }^{16}$ The Jadad scale was used, which contains 2 questions each for randomization and masking and 1 question evaluating the reporting of withdrawals and dropouts. Each question entailed yes or no response options. In total, 5 points were awarded, with a score of 3 or more indicating superior quality. ${ }^{13}$ Allocation concealment and Jadad score were assessed by one observer (R.T.).

A checklist of 32 questions was then prepared from the 22 items of the revised CONSORT guidelines ${ }^{1}$ published in 2001. 
TABLE 1. Distribution of consensus scores for all the items in the modified CONSORT checklist

\begin{tabular}{|c|c|c|c|c|}
\hline Item & $n^{*}$ & No description (\%) & Inadequate (\%) & Adequate (\%) \\
\hline 1. Justification for the trial & 64 & 0 & 32.8 & 67.2 \\
\hline 2. Explicit definition of eligibility criteria & 64 & 1.6 & 32.8 & 65.6 \\
\hline $\begin{array}{l}\text { 3. Detailed description of settings/location of } \\
\text { recruitment and data collection }\end{array}$ & 64 & 0 & 92.2 & 7.8 \\
\hline 4. Details of intervention studied & 64 & 0 & 73.4 & 26.6 \\
\hline 5. Clear statement of hypothesis or objectives & 64 & 0 & 7.8 & 92.2 \\
\hline 6. Identification and definition of outcome measures & 64 & 0 & 23.4 & 76.6 \\
\hline 7. Description of prestudy sample size calculation & 64 & 64.1 & 15.6 & 20.3 \\
\hline $\begin{array}{l}\text { 8. Description of the generation of unpredictable } \\
\text { allocation sequence }\end{array}$ & 64 & 78.1 & 1.6 & 20.3 \\
\hline 9. Details of any restriction used in randomization & 64 & 65.6 & 12.5 & 21.9 \\
\hline 10. Description of allocation concealment & 64 & 85.9 & 9.4 & 4.7 \\
\hline $\begin{array}{l}\text { 11. Details of personnel involved in sequence allocation, } \\
\text { enrollment, and assignment }\end{array}$ & 64 & 92.2 & 7.8 & 0 \\
\hline 12. Details of blinding of participants (where practical) & 53 & 60.4 & 7.5 & 21.1 \\
\hline $\begin{array}{l}\text { 13. Details of blinding of treatment providers (where } \\
\text { practical) }\end{array}$ & 30 & 56.7 & 10.0 & 33.3 \\
\hline 14. Details of blinding of assessors & 64 & 51.6 & 10.9 & 37.5 \\
\hline 15. Details of blinding of analysts & 64 & 98.4 & 1.6 & 0 \\
\hline 16. Details of measurement of success of blinding & 64 & 100 & 0 & 0 \\
\hline 17. Description of statistical methods & 64 & 1.6 (inadequate) & 87.5 (minor errors) & 10.9 (no obvious errors) \\
\hline $\begin{array}{l}\text { 18. Flow chart describing patient numbers at different } \\
\text { stages }\end{array}$ & 64 & 96.9 & 1.6 & 1.6 \\
\hline 19. Clear description of protocol deviations & 30 & 0 & 0 & 100 \\
\hline 20. Description of dates of recruitment & 64 & 40.6 & 9.4 & 50 \\
\hline 21. Details of follow-up & 31 & 9.7 & 22.6 & 67.7 \\
\hline 22. Description of baseline characteristics & 64 & 1.6 & 7.8 & 90.0 \\
\hline 23. Reporting of intention-to-treat principle & 60 & 31.7 & 0 & 68.3 \\
\hline 24. Complete reporting of results & 64 & 0 & 10.9 & 89.1 \\
\hline 25. Reporting of confidence intervals & 64 & 84.4 & 9.4 & 6.3 \\
\hline 26. Multiple testing and corrections & 47 & 74.5 & 10.6 & 14.9 \\
\hline 27. Description of side effects/adverse events & 53 & 9.4 & 18.9 & 71.7 \\
\hline 28. Trial limitations and weaknesses & 64 & 23.4 & 40.6 & 35.9 \\
\hline 29. External validity of trial results & 64 & 0 & 79.7 & 20.3 \\
\hline 30. Literature review & 64 & 0 & 48.4 & 51.6 \\
\hline
\end{tabular}

CONSORT, Consolidated Standards for Reporting of Trials. *Denominator 64 trials, except where an item was not applicable for particular trials.

The score for each item ranged from 1 to 3 (1, no description; 2, inadequate description; 3 , adequate description). The CONSORT guidelines were studied in depth by the reviewers, and the definition of each checklist item was discussed. The checklist was then piloted independently by 3 observers (R.T., S.P.B., and D.E.) on 4 RCTs (published before 2003) to address any ambiguous questions and questions that might yield unusable data. After this, a final checklist involving 30 questions (Table 1) was accepted for characterization of RCTs, excluding the first item (title and abstract, because the authors have to follow the instructions of the journal in preparing the abstract) of the CONSORT guidelines. Each article included in the study was then assessed for every item on the checklist and scored independently by 2 observers (R.T. and S.P.B.) to arrive at a consensus-modified CONSORT score. Discrepancies between the 2 observers were resolved by the third observer (D.E.). The scores for the 30 items were added, and a percentage score was calculated for each trial (some items were not applicable and did not merit any score).
A questionnaire was designed to ascertain the awareness of the CONSORT statement, the views of authors on endorsement of the CONSORT statement by cardiothoracic journals, the possible improvement of reporting quality if the cardiothoracic journals endorse the CONSORT statement, and the difficulties (if any) of conducting RCTs in cardiothoracic surgery. The corresponding authors of all the potentially included RCTs $(n=75)$ published in the 4 principal cardiothoracic surgical journals (in which the CONSORT statement was not endorsed) during the study period were contacted initially by e-mail. Nonresponders were contacted twice again by e-mail, followed by a postal questionnaire with a self-addressed envelope and finally by telephone (wherever the telephone numbers were available).

The data were collected on an Excel spreadsheet (Microsoft Corp, Redmond, Wash) and exported to SPSS (version 12.0 for Windows; SPSS Inc, Chicago, Ill) for analysis. Initial presentations were descriptive, which included the median (interquartile range) Jadad scores, modified CONSORT scores, and percentages 
TABLE 2. Characteristics of RCTs published in the different medical and surgical journals

\begin{tabular}{|c|c|c|c|c|c|c|}
\hline Journal & $\begin{array}{l}\text { Impact factor } \\
\text { in } 2002\end{array}$ & $\begin{array}{l}\text { No. } \\
\text { articles }\end{array}$ & $\begin{array}{c}\text { CONSORT } \\
\text { endorsement }\end{array}$ & $\begin{array}{l}\text { Jadad score, } \\
\text { median (IOR) }\end{array}$ & $\begin{array}{c}\text { Modified } \\
\text { CONSORT score, } \\
\text { median (IOR) }\end{array}$ & $\begin{array}{c}\text { Adequate } \\
\text { reporting of } \\
\text { allocation } \\
\text { concealment }(\%)^{*}\end{array}$ \\
\hline \multicolumn{7}{|l|}{ Surgical journals } \\
\hline Annals of Thoracic Surgery & 2.052 & 26 & No & $2(1-2.25)$ & $65.5(63.9-67.8)$ & 23 \\
\hline $\begin{array}{l}\text { Journal of Thoracic and Cardiovascular } \\
\text { Surgery }\end{array}$ & 2.842 & 26 & No & $2(1-3)$ & $67.3(60.6-75.8)$ & 27 \\
\hline $\begin{array}{l}\text { European Journal of Cardiothoracic } \\
\text { Surgery }\end{array}$ & 1.451 & 10 & No & $2(1-2)$ & $62.4(61.3-65.7)$ & 0 \\
\hline $\begin{array}{l}\text { Asian Cardiovascular \& Thoracic Annals } \\
\text { Medical journals }\end{array}$ & - & 0 & No & - & - & - \\
\hline $\begin{array}{l}\text { Journal of the American Medical } \\
\text { Association }\end{array}$ & 16.8 & 0 & Yes & - & - & - \\
\hline New England Journal of Medicine & 31.736 & 2 & Yes & $2(1-3)$ & $74.9(73.3-76.5)$ & 50 \\
\hline British Medical Journal & 7.585 & 0 & Yes & - & - & - \\
\hline Lancet & 15.397 & 0 & Yes & - & - & - \\
\hline Overall scores & & 64 & & $2(1-3)$ & $65.5(61.7-70.2)$ & 22 \\
\hline
\end{tabular}

RCT, Randomized controlled trial; CONSORT, Consolidated Standards for Reporting of Trials; IQR, interquartile range. *Assessment adapted from Schulz and colleagues. ${ }^{16}$

of the RCT reports reporting allocation concealment adequately of the individual journals and the specific characteristics of the studies. The association between study characteristics (number of authors, single center or multicenter, statistician/epidemiologist involvement, and funding source) and the awareness of authors about the CONSORT statement with the modified CONSORT score (measured as greater or less than the median) was studied. The responses of the authors on the views of conducting and reporting of RCTs were presented as percentages. Nonparametric tests were used for all analyses.

\section{Results}

The proportion of RCTs in principal cardiothoracic journals is approximately $6 \%$, as compared with $12 \%$ in the general medical journals included in this study. Of the 81 articles that were initially retrieved from the 8 different journals ( 4 surgical [75 articles] and 4 general medical [6 articles]), 64 (62 from surgical and 2 from medical journals) were eligible for the final analysis in the study. The reasons for exclusion were as follows: trials with predominantly nonclinical outcomes $(n=5)$, previous reporting of study results $(n=4)$, not truly randomized $(n=2)$, noncardiothoracic surgical topics $(\mathrm{n}=1)$, cluster-randomized trials $(\mathrm{n}=1)$, and studies reporting trial design only $(n=1)$. Three studies for which design and methods had been reported before 2001 were excluded but were subsequently included in sensitivity analyses.

Table 2 shows the different journals along with their impact factor for 2002, number of articles analyzed $(\mathrm{n}=$ 64), endorsement of the CONSORT statement in their "Instructions to Authors" section, median Jadad score, and median modified CONSORT score, calculated as percent- age and percentage of articles reporting allocation concealment. The agreement of the pair of observers who independently assessed the RCTs by using the CONSORT checklist was good (intraclass correlation coefficient, $0.85 ; 95 \%$ confidence interval, $0.76-0.90 ; P<.001)$. The 3 qualityassessment tools used in this study (Jadad score, allocation concealment, and modified CONSORT score) correlated moderately with each other (Jadad and allocation concealment: Spearman rho, 0.57; $P<.001$; Jadad and modified CONSORT: Spearman rho, 0.56; $P<.001$; allocation concealment and modified CONSORT: Spearman rho, 0.47; $P<.001)$.

Table 3 shows the study characteristics (including the number of authors, involvement of a statistician/epidemiologist, single-center or multicenter study, and funding source) of all the RCTs included in the analysis. Because these factors did not differ between the 62 RCTs in the cardiothoracic journals and the 2 from the general journals, further analyses were based on all 64 trials.

Table 1 shows the distribution of scores on all the items on the CONSORT checklist based on all 64 trials except where an item was not applicable (denominators are shown in Table 1). Univariate analysis was performed to determine the association of trial reporting quality (measured as greater or less than the median modified CONSORT score of 65). Parameters included author numbers, statistician/ epidemiologist involvement, number of centers, and funding source. The reporting quality was not significantly associated with studies with higher author numbers (MannWhitney $U$ test: $z=-0.829 ; P=.407)$, multicenter studies 
TABLE 3. Characteristics of the RCTs studied

\begin{tabular}{lc}
\hline Characteristic assessed & $\begin{array}{c}\text { No. }(\%) \text { of trials } \\
(\mathbf{n}=64)\end{array}$ \\
\hline Authors & \\
$\quad<6$ & $16(25)$ \\
$6-10$ & $40(62.5)$ \\
$>10$ & $8(12.5)$ \\
Statistician/epidemiologist involvement & \\
$\quad$ No mention & $45(70.3)$ \\
Involvement acknowledged & $9(14.1)$ \\
Involved as coauthor & $10(15.6)$ \\
Number of centers & \\
Single center & $58(90.6)$ \\
Multicenter & $6(9.4)$ \\
Funding source & \\
No mention & $38(59.4)$ \\
Commercial & $7(10.9)$ \\
Public sector & $15(23.4)$ \\
Mixed & $3(4.7)$ \\
Personal & $1(1.6)$ \\
Country & \\
United States & $13(20.3)$ \\
Europe & $31(48.4)$ \\
Others & $17(26.6)$ \\
Spanning continents & $3(4.7)$ \\
\hline
\end{tabular}

RCT, Randomized controlled trial.

(Fisher exact test: $P=.074$ ), studies with a declared funding source $\left(\chi^{2}\right.$ test with Yates correction: $\chi^{2}=0.117 ; P=$ .732), or involvement of a statistician/epidemiologist in the study (Fisher exact test: $P=.091$ ). Multicenter studies span- ning across continents were associated with a higher-thanaverage quality of reporting (Kruskal-Wallis test: $P=.005$ ). Interpretation of this finding should be made with caution because of the small number $(n=3)$ of studies included in this group.

A sensitivity analysis was conducted to include the 3 studies that had originally been excluded because their design and methods were reported before 2001. The overall median modified CONSORT Score $(65.5 ; 61.7-71.3)$, median Jadad Score (2; 1-3), and percentage of articles reporting adequate allocate concealment (23.8) for the 67 studies did not differ significantly from the analysis of the original 64 included studies.

Authors of 52 RCTs (69\% of the 75 RCTs in principal cardiothoracic journals) responded to the survey about the CONSORT statement by the cardiothoracic journals (Table 4). Nineteen (37\%) were aware of CONSORT; half of the responders thought that cardiothoracic journals should endorse CONSORT, and the opinion of $28(54 \%)$ was that this may improve reporting quality, although only 3 thought this would have meant reporting their trial differently. Three quarters of the respondents thought RCTs were difficult to conduct in surgical specialties.

The articles of 42 of the 52 survey respondents were assessed for their reporting quality. There was no significant difference in the reporting quality between trials whose authors were aware of the CONSORT statement $(\mathrm{n}=15)$ and those whose authors were not $(\mathrm{n}=27$; Mann-Whitney $U$ test: $z=-1.709 ; P=.087$ ).

TABLE 4. Awareness of the CONSORT statement and the views of authors reporting randomized controlled trials in principal cardiothoracic surgical journals during the study period

\begin{tabular}{|c|c|c|c|}
\hline Variable & Yes & No & Other \\
\hline Awareness of CONSORT statement $(n=52)^{*}$ & $36.5 \%$ & $63.5 \%$ & - \\
\hline $\begin{array}{l}\text { Will endorsement of CONSORT statement by the journals } \\
\text { improve the reporting of RCTs }(n=52) \text { ? }\end{array}$ & $53.8 \%$ & $7.7 \%$ & Can't comment $(38.5 \%)$ \\
\hline $\begin{array}{l}\text { Reporting of the trial in a different way if the journals } \\
\text { endorsed CONSORT statement }(n=52)\end{array}$ & $5.8 \%$ & $53.8 \%$ & Maybe $(40.4 \%)$ \\
\hline $\begin{array}{l}\text { Opinion on whether the cardiothoracic surgery journals } \\
\text { should endorse CONSORT statement }(n=52)\end{array}$ & $51.9 \%$ & $5.8 \%$ & Can't comment $(42.3 \%)$ \\
\hline $\begin{array}{l}\text { Is conducting an RCT more difficult in surgical } \\
\text { specialties than medical specialties }(n=52) ?\end{array}$ & $65.4 \%$ & $24.0 \%$ & Maybe $(9.6 \%)$ \\
\hline \multicolumn{4}{|l|}{$\begin{array}{l}\text { Possible difficulties of conducting RCTs in surgical } \\
\text { specialties }(\mathrm{n}=39) \dagger\end{array}$} \\
\hline Randomization & $48.7 \%$ & $41.0 \%$ & Maybe $(5.1 \%)$ \\
\hline Blinding & $74.3 \%$ & $12.8 \%$ & Maybe $(12.9 \%)$ \\
\hline Funding & $48.7 \%$ & $41.0 \%$ & Maybe $(10.2 \%)$ \\
\hline Ethical issues & $48.7 \%$ & $35.8 \%$ & Maybe $(15.3 \%)$ \\
\hline Sample size & $66.6 \%$ & $15.3 \%$ & Maybe $(17.9 \%)$ \\
\hline Lack of peer group support & $33.3 \%$ & $28.2 \%$ & Maybe $(38.4 \%)$ \\
\hline
\end{tabular}

CONSORT, Consolidated Standards for Reporting of Trials; RCT, randomized controlled trial. *Total number of authors who participated in the survey. $\dagger$ Number of authors who believe that conducting RCTs in surgical specialties is more difficult than in medical specialties. 


\section{Discussion}

It is more than 50 years since the first RCT was reported. ${ }^{17}$ It is estimated that the number of RCTs is increasing by a factor of 10 every year. ${ }^{18}$ The results of RCTs provide the most reliable evidence about which treatments are best for patients. It is therefore important that RCTs be reported in a high-quality manner so readers have a clear view on why the study was conducted, how it was conducted, and how it was analyzed. This would be helpful not only in the immediate appraisal of trials, but also in the long term, when further analyses are performed, such as in systematic reviews and meta-analyses.

Our study shows that the reporting quality of RCTs in cardiothoracic surgery is suboptimal. Among the study characteristics assessed, only multicenter RCTs spanning across continents were found to have an above-average quality of reporting. Although the number of multicontinental reports $(\mathrm{n}=3)$ was small, we speculate that this association (if real) may be due to the involvement of a wide range of experts at the inception and in the oversight of such large studies.

The main purpose of randomization is to eliminate selection bias, which is a crucial component for high-quality RCTs. In addition, randomization aims to create groups that are comparable in terms of any known or unknown potential confounding factors. ${ }^{19}$ Proper randomization includes $2 \mathrm{im}$ portant elements: generation of an unpredictable allocation sequence and concealment of this sequence from the investigators enrolling participants into the study. Unlike blinding, which may not be possible to practice at all times (especially with surgical trials), allocation concealment can always be successfully implemented. ${ }^{4,16}$ The reporting of the generation of unpredictable allocation sequences was adequate in $20.3 \%$ (Table 1) and allocation concealment was adequate in only $22 \%$ of the reports (Table 2) in our study. Trials that have not reported adequate allocation concealment have been found to be associated with exaggerated treatment effects. ${ }^{16}$ The reporting of blinding of participants, treatment providers, and assessors was adequate in $21.1 \%, 33.3 \%$, and $37.5 \%$ of the reports, respectively. Most of the studies reported as "double-blinded" trials did not provide details on who was blinded. Similar exclusion of details on the double blinding was reported by Schulz and associates. ${ }^{20}$ Omitting such details can create confusion and skepticism in interpreting the results. Studies have shown that nonrandomized trials and RCTs that do not incorporate blinding are more likely to show advantages of a new intervention over the standard treatment. ${ }^{21}$ RCTs that have not reported blinding have been shown to exaggerate treatment effects. ${ }^{16}$

One of the important recommendations of the CONSORT statement is to produce a flow diagram that describes the flow of participants through all the stages of trial. This was shown to improve the quality of reporting. ${ }^{22}$ In our study, a flow diagram was shown in only $1.6 \%$ of the reports.

Prestudy sample size calculations based on a clearly defined outcome are considered essential for both scientific and ethical reasons. In our study, reporting of the prestudy sample size calculation was adequate in $20.3 \%$ of the reports. Studies with small sample sizes are often inadequately powered to detect small but clinically significant differences between interventions. ${ }^{23}$

Intention-to-treat analyses are usually favored over perprotocol analyses because they avoid bias associated with nonrandom loss of participants. ${ }^{24}$ Although such analyses may underestimate the real benefit of treatments for which noncompliance may be an issue, they address the effectiveness question, which is the more pragmatic approach to the application of the interventions in clinical practice. Intentionto-treat analyses were reported in $68.3 \%$ of the RCTs in this study.

The reporting of details that would aid in assessing the external validity was poor in most of the RCTs and systematic reviews. Trials lacking the details on external validity can lead to underuse of treatments that are effective. ${ }^{25}$ Details that could be used to assess the external validity in our study were presented in $20.3 \%$ of the trials.

The need for improving the overall reporting of RCTs has been highlighted by several other studies. ${ }^{4,10,11,26} \mathrm{Al}-$ though the reporting of RCTs can give some clues on the quality of the methodology, poor reporting does not necessarily mean that the trials were poorly designed and executed. Well-conducted trials may be reported badly, and studies with poor methodologies can be reported in such as way as to hide important deficiencies. ${ }^{14,27}$

The potential difficulties associated with performing RCTs in surgical practice include the difficulty in successfully blinding patients, investigators, and assessors; the variability of surgical techniques and operator skills; and the learning curve (where the technical performance of a new technique improves over time ${ }^{28}$ ), which influences the efficacy of many interventions under study. In addition, lack of training on the conduct of clinical trials during residencies and fellowships may also be an important element. These may be the reasons why RCTs in surgical specialties are comparatively fewer than in the medical specialties.

Nevertheless, although careful statistical techniques can help to reduce biases in nonrandomized studies, they cannot deal with the major problems of selection bias. ${ }^{29}$ We therefore believe that well-conducted and properly reported RCTs are essential for improving the overall quality of surgical care.

The 2 elements that could potentially improve the reporting of RCTs in cardiothoracic surgery may be awareness of the CONSORT statement among authors and, more important than awareness, endorsement of the CONSORT state- 
ment by the cardiothoracic surgical journals. Although the dissemination of the CONSORT statement in several languages may have improved its awareness, our study shows that more than $60 \%$ of the authors who reported their studies in the principal cardiothoracic journals were not aware of it. This may be because of the publication of the CONSORT statement in general medical journals rather than the specialist surgical journals. Awareness of the statement, however, may not improve the quality of reporting on its own. In our study, awareness of the CONSORT statement among the authors was not significantly associated with improved quality of reporting. There is, however, evidence to suggest that the quality of reporting of RCTs has improved when the journals have endorsed the statement as compared with when the CONSORT statement was not endorsed. ${ }^{15,22}$ The use of the CONSORT statement was also shown to positively influence the manner in which the RCTs were conducted. ${ }^{3}$ The endorsement of the CONSORT statement by the journals may also help in improving the awareness of CONSORT statement because the authors would have to follow the "Instructions for Authors" of the journal in which they would like to publish their study.

In our study, more than $40 \%$ of authors replied that they may have reported their trial differently, and approximately $6 \%$ of authors said that they would have definitely reported their trial differently if the cardiothoracic journals had endorsed the CONSORT statement. As shown in Table 4, more than $50 \%$ of the authors believe that endorsement of the statement by cardiothoracic journals may further improve the reporting of RCTs in cardiothoracic surgery. Whether this belief of improvement in the reporting quality of RCTs in cardiothoracic surgery is true remains to be assessed in future research if the cardiothoracic journals endorse the CONSORT statement.

Our survey shows that most of the authors $(65.4 \%)$ believe that conducting high-quality RCTs is more difficult in surgical specialties than in medical specialties, with the main difficulties including blinding of treatment providers and participants (74.3\%) and obtaining a sufficient sample size to be able to draw conclusions (66.6\%). These findings are similar to the views of Anyanwu and Treasure. ${ }^{11}$ However, this should not deter surgical researchers in conducting RCTs to investigate the problems that could be answered best by this design.

Because of restrictions on resources and to preserve blinding of the reviewers to the source journals of the articles, the initial screening of articles was not performed separately by 2 people. This blinding may not, however, have been completely successful, because the reviewers may have previously encountered some of the articles assessed and could have been prejudiced by preexisting knowledge on the subject. In addition, the CONSORT checklist contained items whose scoring was subjective and dependent on reviewers' perceptions and domain knowledge. The assessment of the item of "allocation concealment" as part of the modified CONSORT checklist was slightly at variance with the assessment described by Schulz and associates ${ }^{16}$ in 1995, which explains the difference in the scoring as shown in Tables 1 and 2. Finally, this study was restricted to cardiothoracic surgery (reflecting the interests of the authors and limitations in our time and other resources). The conclusions are not necessarily generalizable to trials in other areas of surgery or to medical trials.

In conclusion, the results of our study suggest that the reporting quality of RCTs in cardiothoracic surgery is suboptimal. Although studies spanning across continents (where numbers were small) were associated with above-average reporting quality, other study characteristics were not associated with reporting quality. Most of the authors were not aware of the CONSORT statement.

Because the results of RCTs will have a significant effect on clinical decision making at all levels, it is important to improve the quality of reporting. This may be achieved by the endorsement of the CONSORT statement by cardiothoracic journals, as well as increasing the awareness of the CONSORT statement among cardiothoracic surgical researchers.

\section{References}

1. Altman DG, Schulz KF, Moher D, Egger M, Davidoff F, Elbourne D, et al. The revised CONSORT statement for reporting randomized trials: explanation and elaboration. Ann Intern Med. 2001;134:663-94.

2. Juni P, Altman DG, Egger M. Systematic reviews in health care: assessing the quality of controlled clinical trials. BMJ. 2001;323:42-6.

3. Moher D, Schulz KF, Altman DG. The CONSORT statement: revised recommendations for improving the quality of reports of parallelgroup randomised trials. Lancet. 2001;357:1191-4.

4. Schulz KF, Chalmers I, Grimes DA, Altman DG. Assessing the quality of randomization from reports of controlled trials published in obstetrics and gynecology journals. JAMA. 1994;272:125-8.

5. Begg C, Cho M, Eastwood S, Horton R, Moher D, Olkin I, et al. Improving the quality of reporting of randomized controlled trials. The CONSORT statement. JAMA. 1996;276:637-9.

6. Altman DG. Better reporting of randomised controlled trials: the CONSORT statement. BMJ. 1996;313:570-1.

7. Rennie D. How to report randomized controlled trials. The CONSORT statement. JAMA. 1996;276:649.

8. Altman DG. Endorsement of the CONSORT statement by high impact medical journals: survey of instructions for authors. BMJ. 2005;330: 1056-7.

9. Junker CA. Adherence to published standards of reporting: a comparison of placebo-controlled trials published in English or German. JAMA. 1998;280:247-9.

10. Ko CY, Sack J, Chang JT, Fink A. Reporting randomized, controlled trials: where quality of reporting may be improved. Dis Colon Rectum. 2002;45:443-7.

11. Anyanwu AC, Treasure T. Surgical research revisited: clinical trials in the cardiothoracic surgical literature. Eur J Cardiothorac Surg. 2004; 25:299-303.

12. Moher D, Jadad AR, Nichol G, Penman M, Tugwell P, Walsh S. Assessing the quality of randomized controlled trials: an annotated bibliography of scales and checklists. Control Clin Trials 1995;16: $62-73$.

13. Jadad AR, Moore RA, Carroll D, Jenkinson C, Reynolds DJ, Gavaghan DJ, et al. Assessing the quality of reports of randomized clinical trials: is blinding necessary? Control Clin Trials 1996;17:1-12. 
14. Huwiler-Muntener K, Juni P, Junker C, Egger M. Quality of reporting of randomized trials as a measure of methodologic quality. JAMA. 2002;287:2801-4.

15. Moher D, Jones A, Lepage L. Use of the CONSORT statement and quality of reports of randomized trials: a comparative before-and-after evaluation. JAMA. 2001;285:1992-5.

16. Schulz KF, Chalmers I, Hayes RJ, Altman DG. Empirical evidence of bias. Dimensions of methodological quality associated with estimates of treatment effects in controlled trials. JAMA. 1995;273:408-12.

17. Andrews G. Randomised controlled trials in psychiatry: important but poorly accepted. BMJ. 1999;319:562-4.

18. Reizenstein P, Delgado M, Gastiaburu J. Efficacy of and errors in randomized multicenter trials. A review of 230 clinical trials. Biomed Pharmacother. 1983;37:14-24.

19. Altman DG, Bland JM. Statistics notes. Treatment allocation in controlled trials: why randomise? BMJ. 1999;318:1209.

20. Schulz KF, Grimes DA, Altman DG, Hayes RJ. Blinding and exclusions after allocation in randomised controlled trials: survey of published parallel group trials in obstetrics and gynaecology. BMJ. 1996; 312:742-4.
21. Grimes DA. Randomized controlled trials: "it ain't necessarily so." Obstet Gynecol. 1991;78:703-4.

22. Egger M, Juni P, Bartlett C. Value of flow diagrams in reports of randomized controlled trials. JAMA. 2001;285:1996-9.

23. Altman DG, Bland JM. Absence of evidence is not evidence of absence. BMJ. 1995;311:485.

24. Lachin JM. Statistical considerations in the intent-to-treat principle. Control Clin Trials. 2000;21:167-89.

25. Rothwell PM. External validity of randomised controlled trials: "to whom do the results of this trial apply?" Lancet. 2005;365:82-93.

26. Chan AW, Altman DG. Epidemiology and reporting of randomised trials published in PubMed journals. Lancet. 2005;365:1159-62.

27. Soares HP, Daniels S, Kumar A, Clarke M, Scott C, Swann S, et al Bad reporting does not mean bad methods for randomised trials: observational study of randomised controlled trials performed by the Radiation Therapy Oncology Group. BMJ. 2004;328:22-4.

28. Cook JA, Ramsay CR, Fayers P. Statistical evaluation of learning curve effects in surgical trials. Clin Trials. 2004;1:421-7.

29. Dunn G. Estimating the causal effects of treatment. Epidemiol Psichiatr Soc. 2002;11:206-15. 